\title{
Automorphisms of Kronrod-Reeb graphs of Morse functions on 2-sphere
}

\author{
Anna Kravchenko, Sergiy Maksymenko
}

Abstract. Let $M$ be a compact two-dimensional manifold, $f \in C^{\infty}(M, \mathbb{R})$
be a Morse function, and $\Gamma_{f}$ be its Kronrod-Reeb graph. Denote by
\[ \mathcal{O}(f)=\{f \circ h \mid h \in \mathcal{D}\} \] the orbit of $f$ with respect to the natural right action of the group of diffeomorphisms $\mathcal{D}$ on $C^{\infty}(M, \mathbb{R})$, and by $\mathcal{S}(f)=\{h \in \mathcal{D} \mid f \circ h=f\}$ the corresponding stabilizer of this function. It is easy to show that each $h \in \mathcal{S}(f)$ induces a homeomorphism of $\Gamma_{f}$. Let also $\mathcal{D}_{\text {id }}(M)$ be the identity path component of $\mathcal{D}(M), \mathcal{S}^{\prime}(f)=\mathcal{S}(f) \cap \mathcal{D}_{\text {id }}(M)$ be group of diffeomorphisms of $M$ preserving $f$ and isotopic to identity map, and $G_{f}$ be the group of homeomorphisms of the graph $\Gamma_{f}$ induced by diffeomorphisms belonging to $\mathcal{S}^{\prime}(f)$. This group is one of the key ingredients for calculating the homotopy type of the orbit $\mathcal{O}(f)$.

Recently, the authors described the structure of groups $G_{f}$ for Morse functions on all orientable surfaces distinct from 2-torus and 2-sphere. The present paper is devoted to the case $M=S^{2}$. In this situation $\Gamma_{f}$ is always a tree, and therefore all elements of the group $G_{f}$ have a common fixed subtree $\operatorname{Fix}\left(G_{f}\right)$, which may even consist of a unique vertex. Our main result calculates the groups $G_{f}$ for all Morse functions $f: S^{2} \rightarrow \mathbb{R}$ whose fixed subtree $\operatorname{Fix}\left(G_{f}\right)$ consists of more than one point.

Анотація. Нехай $M$ - компактна двовимірна поверхня і $f: M \rightarrow \mathbb{R}-$ функція Морса і $\Gamma_{f}$ — ï̈ граф Кронрода-Ріба. Визначимо природну праву дію групи $\mathcal{C}^{\infty}$ дифеоморфізмів $\mathcal{D}(M)$ поверхні $M$ на $\mathcal{C}^{\infty}(M, \mathbb{R})$ за правилом: результат дії дифеоморфізма $h \in \mathcal{D}(M)$ на $f \in \mathcal{C}^{\infty}(M, \mathbb{R})$ це композиція $f \circ h: M \rightarrow \mathbb{R}$. Для $f \in \mathcal{C}^{\infty}(M, \mathbb{R})$ позначимо через $\mathcal{O}(f)=\{f \circ h \mid h \in \mathcal{D}(M)\}$ та $\mathcal{S}(f)=\{h \in \mathcal{D}(M) \mid f \circ h=f\}$ - відповідно орбіту та стабілізатор цієї функції. Легко показати, що кожен $h \in \mathcal{S}(f)$ індукує деякий гомеоморфізм $\rho(h)$ графа $\Gamma_{f}$, а відповідність $h \mapsto \rho(h)$ є гомоморфізмом з $\mathcal{S}(f)$ в групу гомеоморфізмів $\Gamma_{f}$. Нехай $\mathcal{D}_{\mathrm{id}}(M)-$ тотожна компонента зв'язності групи $\mathcal{D}(M), \mathcal{S}^{\prime}(f)=\mathcal{S}(f) \cap \mathcal{D}_{\text {id }}(M)-$ підгрупа в $\mathcal{D}_{\text {id }}(M)$ що складається з дифеоморфізмів які зберігають $f$

2010 Mathematics Subject Classification: 37E30, 22F50

Keywords: Morse function, Kronrod-Reeb graph

DOI: http://dx.doi.org/ 
i орієнтацію $M$ та ізотопні до тотожного відображення, і $G_{f}$ - група автоморфізмів графа Кронрода-Ріба функції $f$ індукованих дифеоморфізмами з $\mathcal{S}^{\prime}(f)$. Остання група є скінченною і відіграє ключову роль для обчислення гомотопічного типу орбіти $\mathcal{O}(f)$.

В попередній статті автори описали алгебраїчну структуру груп $G_{f}$ для функцій Морса на всіх орієнтовних поверхнях відмінних від тора $T^{2}$ та 2-сфери $S^{2}$. Дана робота присвячена випадку $M=S^{2}$. Відмітимо, що для кожної функції Морса $f: S^{2} \rightarrow \mathbb{R}$ їі граф $\Gamma_{f}$ завжди є деревом, а тому всі елементи групи $G_{f}$ мають спільне нерухому піддерево $\operatorname{Fix}\left(G_{f}\right)$, яке може складатись з однієї вершини. Основний результат даної статті обчислює групи $G_{f}$ для всіх функцій Морса $f: S^{2} \rightarrow \mathbb{R}$, у яких нерухоме піддерево $\operatorname{Fix}\left(G_{f}\right)$ містить більше ніж одну точку.

Отримані результати мають місце також для більш широкого ніж морсівські класу функцій $f: S^{2} \rightarrow \mathbb{R}$, що є гладко еквівалентними до однорідних многочленів без кратних множників в околі кожної своєї критичної точки.

\section{INTRODUCTION}

Let $M$ be a compact two-dimensional manifold and $\mathcal{D}(M)$ the group of diffeomorphisms of $M$. Then there exists a natural right action

$$
\phi: \mathcal{C}^{\infty}(M, \mathbb{R}) \times \mathcal{D}(M) \rightarrow \mathcal{C}^{\infty}(M, \mathbb{R})
$$

of this group on the space of smooth functions on $M$ defined by the formula $\phi(f, h)=f \circ h$. For $f \in \mathcal{C}^{\infty}(M, \mathbb{R})$ denote by

$$
\mathcal{S}(f)=\{h \in \mathcal{D}(M) \mid f \circ h=f\}
$$

its stabilizer with respect to the specified action.

Definition 1.1. Let $\mathcal{F}(M, \mathbb{R})$ be the subset of $\mathcal{C}^{\infty}(M, \mathbb{R})$ consisting of maps $f: M \rightarrow \mathbb{R}$ such

(1) $f$ takes constant values on the connected components of the boundary $\partial M$ and has no critical points on $\partial M$;

(2) for each critical point $z$ of $f$ there are local coordiantes $(x, y)$ in which $z=(0,0)$ and $f(x, y)=f(z)+g_{z}(x, y)$, where $g_{z}: \mathbb{R}^{2} \rightarrow \mathbb{R}$ is a homogeneous polynomial without multiple factors.

Notice that every critical point of $f \in \mathcal{F}(M, \mathbb{R})$ is isolated.

A function $f \in \mathcal{F}(M, \mathbb{R})$ is called Morse, if $\operatorname{deg} g_{z}=2$ for each critical point $z$ of $f$. In that case, due to Morse Lemma, one can assuse that $g_{z}(x, y)= \pm x^{2} \pm y^{2}$.

We will denote by $\mathcal{M}(M, \mathbb{R})$ the space of all Morse maps $M \rightarrow \mathbb{R}$.

Homotopy types of stabilizers and orbits of Morse functions and functions from $\mathcal{F}(M, \mathbb{R})$ were studied in [8], [9], [10], [1], [2], [3], [4], [5], [6]. 
Let $f \in \mathcal{C}^{\infty}(M, \mathbb{R}), \Gamma_{f}$ be a partition of the surface $M$ into the connected components of level sets of this function, and $p: M \rightarrow \Gamma_{f}$ be the canonical factor-mapping, associating to each $x \in M$ the connected component of the level set $f^{-1}(f(x))$ containing that point.

Endow $\Gamma_{f}$ with the factor topology with respect to the mapping $p$ : so a subset $A \subset \Gamma_{f}$ will be regarded as open if and only if its inverse image $p^{-1}(A)$ is open in $M$. Then $f$ induces the function $\hat{f}: \Gamma_{f} \rightarrow \mathbb{R}$, such that $f=\hat{f} \circ p$.

It is well known, that if $f \in \mathcal{F}(M, \mathbb{R})$, then $\Gamma_{f}$ has a structure of a onedimensional CW-complex called the Kronrod-Reeb graph, or simply the graph of $f$. The vertices of this graph correspond to critical connected components of level sets of $f$ and connected components of the boundary of the surface. By the edge of $\Gamma_{f}$ we will mean an open edge, that is, a one-dimensional cell.

Denote by $\mathcal{H}\left(\Gamma_{f}\right)$ the group of homeomorphisms of $\Gamma_{f}$. Notice that each element of the stabilizer $h \in \mathcal{S}(f)$ leaves invariant each level set of $f$, and therefore induces a homeomorphism $\rho(h)$ of the graph of $f$, so that the following diagram is commutative:

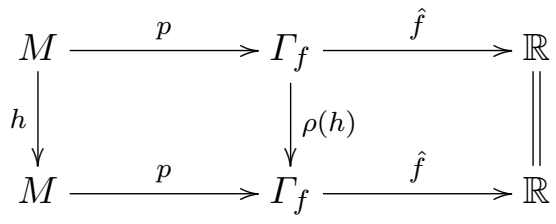

Moreover, the correspondence $h \mapsto g(h)$ is a homomorphism of groups $\rho: \mathcal{S}(f) \rightarrow \mathcal{H}\left(\Gamma_{f}\right)$.

Let also $\mathcal{D}_{\text {id }}(M)$ be the path component of the identity $\operatorname{map} \operatorname{id}_{M}$ in $\mathcal{D}(M)$. Put

$$
\mathcal{S}^{\prime}(f)=\mathcal{S}(f) \cap \mathcal{D}_{\text {id }}(M) \quad G_{f}=\rho\left(\mathcal{S}^{\prime}(f)\right) .
$$

Thus, $G_{f}$ is the group of automorphisms of the Kronrod-Reeb graph of $f$ induced by diffeomorphisms of the surface preserving the function and isotopic identity.

Remark 1.2. Since $\hat{f}$ is monotone on edges of $\Gamma_{f}$, it is easy to show that $G_{f}$ is a finite group. Moreover, if $g(E)=E$, for some $g \in G$ and an edge $E$ of the graph $\Gamma_{f}$, then $g(x)=x$ for all $x \in E$.

Since $G_{f}$ is finite and $\rho$ is continuous, it follows that $\rho$ reduces to an epimorphism $\rho_{0}: \pi_{0} \mathcal{S}^{\prime}(f) \rightarrow G_{f}$ of the group $\pi_{0} \mathcal{S}^{\prime}(f)$ path components of $\mathcal{S}^{\prime}(f)$ being an analogue of the mapping class group for $f$-preserving diffeomorphisms. 
Algebraic structure of the group $\pi_{0} \mathcal{S}^{\prime}(f)$ of connected components of $\mathcal{S}^{\prime}(f)$ for all $f \in \mathcal{F}(M, \mathbb{R})$ on orientable surfaces $M$ distinct from 2-torus and 2-sphere is described in [11], and the structure of its factor group $G_{f}$ is investigated in [7]. These groups play an important role in computing the homotopy type of the path component $\mathcal{O}_{f}(f)$ of the orbit of $f$, see also [8], [9], [1], [2], [3].

The purpose of this note is to describe the groups $G_{f}$ for a certain class of smooth functions on 2-sphere.

The main result Theorem 1.4 reduces computation of $G_{f}$ to computations of similar groups for restrictions of $f$ to some disks in the 2-sphere. As noted above the latter calculations were described in [7].

First we recall a variant of the well known fact about automorphisms of finite trees from graphs theory.

Lemma 1.3. Let $\Gamma$ be a finite contractible one-dimensional $C W$-complex («a topological tree»), $G$ be a finite group of its cellular homeomorphisms, and $\operatorname{Fix}(G)$ be the set of common fixed points of all elements of the group $G$. Then $\operatorname{Fix}(G)$ is either a contractible subcomplex or consists of a single point belonging to some edge $E$ an open 1-cell), and in the latter case there exists $g \in G$ such that $g(E)=E$ and $g$ changes the orientation of $E$.

Suppose $f: \mathcal{S}^{2} \rightarrow \mathbb{R}$ belongs to $\mathcal{F}\left(S^{2}, \mathbb{R}\right)$. Then it is easy to show that $\Gamma_{f}$ is a tree, i.e., a finite contractible one-dimensional $\mathrm{CW}$-complex, and by Remark $1.2 G_{f}$ is a finite group of cellular homeomorphisms of $\Gamma_{f}$. Therefore, for $G_{f}$, the conditions of Lemma 1.3 are satisfied. Note that according to Remark 1.2 the second case of Lemma 1.3 is impossible, and hence $G_{f}$ has a fixed subtree.

In this paper we consider the case when the fixed subtree of the group $G_{f}$ contains more than one vertex, i.e. has at least one edge.

Let us also mention that $\mathcal{D}_{\text {id }}\left(S^{2}\right)$ coincides with the group $\mathcal{D}^{+}\left(S^{2}\right)$ of diffeomorphisms of the sphere preserving orientation, [12]. Therefore $\mathcal{S}^{\prime}(f)$ consists of diffeomorphisms of the sphere preserving the function $f$ and the orientation of $\mathcal{S}^{2}$.

Theorem 1.4. Let $f \in \mathcal{F}\left(S^{2}, \mathbb{R}\right)$. Suppose that all elements of the group $G_{f}$ have a common fixed edge $E$. Let $x \in E$ be an arbitrary point and $A$ and $B$ be the closures of the connected components of $S^{2} \backslash p^{-1}(x)$, see Figure 1.1. Then

(1) $A$ and $B$ are 2-disks being invariant with respect to $\mathcal{S}^{\prime}(f)$;

(2) the restrictions $\left.f\right|_{A} \in \mathcal{F}(A, \mathbb{R})$ and $\left.f\right|_{B} \in \mathcal{F}(B, \mathbb{R})$;

(3) the map $\phi: G_{f} \rightarrow G_{\left.f\right|_{A}} \times G_{\left.f\right|_{B}}$ defined by $\phi(\gamma)=\left(\left.\gamma\right|_{\Gamma_{A}},\left.\gamma\right|_{\Gamma_{B}}\right)$ is an isomorphism of groups. 


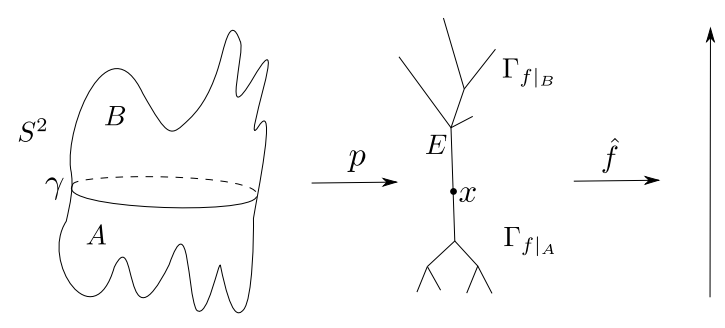

FiguRE 1.1.

Corollary 1.5. For any $a, b \in \mathcal{F}\left(D^{2}, \mathbb{R}\right)$ there exists $f \in \mathcal{F}\left(S^{2}, \mathbb{R}\right)$ such that $G_{f}$ has an fixed subtree consisting of more than one vertex and

$$
G_{f} \cong G_{a} \times G_{b}
$$

Sketch of proof. One can assume that $a$ takes maximal value on $\partial D^{2}$, while $b$ takes a minimal value on $\partial D^{2}$. Now regard $S^{2}=\left\{x^{2}+y^{2}+z^{2}=1\right\}$ as the unit sphere in $\mathbb{R}^{3}$. Let also $A=S^{2} \cap\{z \leq 0\}$ and $B=S^{2} \cap\{z \geq 0\}$ be the lower and upper hemispheres respectively. Then one can assume that the function $a$ is defined on $A$, the function $b$ is defined on $B$, they coincide on the big circle $A \cap B$, and define a function $f \in \mathcal{F}\left(S^{2}, \mathbb{R}\right)$. It then easily follows that $G_{f}$ has a fixed edge containing the point corresponding to $A \cap B$, whence by Theorem 1.4 we get an isomorphism $G_{f} \cong G_{a} \times G_{b}$. We leave the details for the reader.

For the structure of the groups $G_{f}$ for $f \in \mathcal{F}\left(D^{2}, \mathbb{R}\right)$ we refer the reader to the paper [7].

\section{Proof of Theorem 1.4}

(1) By assumption $x$ belongs to the open edge $E$. Therefore $p^{-1}(x)$ is a regular connected component of some level set of the function $f$, that is, a simple closed curve. Then, by Jordan Theorem, $p^{-1}(x)$ divides the sphere into two connected components whose closures are homeomorphic to twodimensional disks. Consequently, $A$ and $B$ are two-dimensional disks.

Let as show that $A$ and $B$ are invariant with respect to $\mathcal{S}^{\prime}(f)$, i.e., $h(A)=A$ and $h(B)=B$ for each $h \in \mathcal{S}^{\prime}(f)$. Denote

$$
\Gamma_{A}=p(A) \quad \Gamma_{B}=p(B) .
$$

Then

$$
\Gamma_{A} \cup \Gamma_{B}=\Gamma \quad \Gamma_{A} \cap \Gamma_{B}=\{x\} .
$$


By definition, $\rho(h)(x)=x$, whence $\rho(h)$ either preserves both $\Gamma_{A}$ and $\Gamma_{B}$ or interchange them. We claim that

$$
\rho(h)\left(\Gamma_{A}\right)=\Gamma_{A} \quad \rho(h)\left(\Gamma_{B}\right)=\Gamma_{B} .
$$

Indeed suppose $\rho(h)\left(\Gamma_{A}\right)=\Gamma_{B}$. Since $\rho(h)$ is fixed on $E$, it follows that

$$
\rho(h)\left(\Gamma_{A} \cap E\right)=\Gamma_{A} \cap E,
$$

whence

$$
\rho(h)\left(\Gamma_{A} \cap E\right)=\rho(h)\left(\Gamma_{A}\right) \cap \rho(E)=\Gamma_{B} \cap E \neq \Gamma_{A} \cap E,
$$

which contradicts to our assumption. Thus $\Gamma_{A}$ and $\Gamma_{B}$ are invariant with respect to the group $G_{f}$.

Now we can show that $A$ and $B$ are also invariant with respect to $h$. By virtue of the commutativity of the diagram (1.1) $\rho(h)(p(y))=p(h(y))$ for all $y \in \Gamma$. In particular:

$$
p(h(A))=\rho(h)(p(A))=\rho(h)\left(\Gamma_{A}\right)=\Gamma_{A} .
$$

Therefore, $h(A)=p^{-1}\left(\Gamma_{A}\right)=A$. The proof for $B$ is similar. Thus, $A$ and $B$ are invariant with respect to $\mathcal{S}^{\prime}(f)$.

(2) Notice that the function $f$ takes a constant value on the simple closed curve $p^{-1}(x)$ being a common boundary of disks $A$ and $B$, and does not contain critical points of $f$. Therefore, the restrictions $\left.f\right|_{A},\left.f\right|_{B}$ satisfy the conditions 1) and 2) the Definition 1.1, and so they belong to $\mathcal{F}(A, \mathbb{R})$ and $\mathcal{F}(B, \mathbb{R})$ respectively.

(3) We should prove that the map $\phi: G_{f} \rightarrow G_{\left.f\right|_{A}} \times G_{\left.f\right|_{B}}$ defined by formula $\phi(\gamma)=\left(\left.\gamma\right|_{\Gamma_{A}},\left.\gamma\right|_{\Gamma_{B}}\right)$ is an isomorphism.

First we will show that $\phi$ is correctly defined. Let $\gamma \in G_{f}=\rho\left(\mathcal{S}^{\prime}(f)\right)$, that is, $\gamma=\rho(h)$, where $h$ is a diffeomorphism of the sphere preserving the function $f$ and isotopic to the identity.

We claim that $\left.h\right|_{A} \in \mathcal{S}^{\prime}\left(\left.f\right|_{A}\right)=\mathcal{S}\left(\left.f\right|_{A}\right) \cap \mathcal{D}_{\text {id }}(A)$. Indeed, for each point $x \in A$ we have that:

$$
f(x)=\left.f\right|_{A}(x)=\left.f\right|_{A}\left(\left.h\right|_{A}(x)\right)=\left.f\right|_{A}(h(x))=f(h(x)),
$$

which means that $\left.h\right|_{A} \in \mathcal{S}\left(\left.f\right|_{A}\right)$.

Moreover, since $h$ preserves the orientation of the sphere, it follows that $\left.h\right|_{A}$ preserves the orientation of the disk $A$, whence by [12], $\left.h\right|_{A} \in \mathcal{D}_{\text {id }}(A)$. Thus $\left.\gamma\right|_{\Gamma_{A}} \in G_{\left.f\right|_{A}}$. Similarly $\left.\gamma\right|_{\Gamma_{B}} \in G_{\left.f\right|_{B}}$, and so $\phi$ is well defined.

Let us now verify that $\phi$ is an isomorphism of groups, that is, a biective homomorphism. Indeed, if $\delta, \omega \in G_{f}$, then

$$
\begin{aligned}
\phi(\delta \circ \omega) & =\left(\left.\delta \circ \omega\right|_{\Gamma_{A}},\left.\delta \circ \omega\right|_{\Gamma_{B}}\right)= \\
& =\left(\left.\delta\right|_{\Gamma_{A}},\left.\delta\right|_{\Gamma_{B}}\right) \circ\left(\left.\omega\right|_{\Gamma_{A}},\left.\omega\right|_{\Gamma_{B}}\right)=
\end{aligned}
$$




$$
\begin{aligned}
& =\left(\left.\left.\delta\right|_{\Gamma_{A}} \circ \omega\right|_{\Gamma_{A}},\left.\left.\delta\right|_{\Gamma_{B}} \circ \omega\right|_{\Gamma_{B}}\right)= \\
& =\left(\left.\delta \circ \omega\right|_{\Gamma_{A}},\left.\delta \circ \omega\right|_{\Gamma_{B}}\right),
\end{aligned}
$$

sp $\phi$ is a homomorphism.

Let us show that $\operatorname{ker} \phi=\left\{\operatorname{id}_{\Gamma}\right\}$. Indeed, suppose $\gamma \in \operatorname{ker} \phi$, that is $\left.\gamma\right|_{\Gamma_{A}}=\operatorname{id}_{\Gamma_{A}}$ and $\left.\gamma\right|_{\Gamma_{B}}=\operatorname{id}_{\Gamma_{B}}$. Then $\gamma$ is fixed on $\Gamma_{A} \cup \Gamma_{B}=\Gamma$, and hence it is the identity map.

Surjectivity of $\phi: G_{f} \rightarrow G_{\left.f\right|_{A}} \times G_{\left.f\right|_{B}}$ is implied by the following simple lemma.

Lemma 2.1. Let $f \in \mathcal{F}(M, \mathbb{R})$. Then for each $\alpha \in G_{f}$, there exists $a \in \mathcal{S}^{\prime}(f)$ fixed near the boundary $\partial M$ and such that $\alpha=\rho(a)$.

Proof. By assumption $\alpha=\rho(h)$ for some $h \in \mathcal{S}^{\prime}(f)=\mathcal{S}(f) \cap \mathcal{D}_{\text {id }}(M)$. Since $h$ is isotopic to $\mathrm{id}_{M}$, it leaves invariant each connected component $V$ of $\partial M$ and preserves its orientation. In particular, the restriction $\left.h\right|_{V}: V \rightarrow V$ is isotopic to $\mathrm{id}_{V}$. As $f$ takes constant value on $V$ and has not critical points near $V, h$ can be deformed in $\mathcal{S}(f)$ to a diffeomorphism $a$ fixed near $\partial M$, e.g. [8, Lemma 4.14], whence $\rho(a)=\rho(h)=\alpha$.

Let $(\alpha, \beta) \in G_{\left.f\right|_{A}} \times G_{\left.f\right|_{B}}$, then by Lemma 2.1 there exist $a \in \mathcal{S}^{\prime}\left(\left.f\right|_{A}\right)$ and $b \in \mathcal{S}^{\prime}\left(\left.f\right|_{B}\right)$ fixed near $\partial A=\partial B=p^{-1}(x)$ and such that $\alpha=\rho_{A}(a)$ and $\beta=\rho_{B}(b)$. Define $h$ by the following formula:

$$
h= \begin{cases}a(x), & x \in A, \\ b(x), & x \in B .\end{cases}
$$

Then, $h$ is a diffeomorphism of the sphere, preserving the function and orientation, whence $h \in \mathcal{S}^{\prime}(f)$.

Moreover if we put $\gamma=\rho(h) \in G_{f}$, then $\left.\gamma\right|_{\Gamma_{A}}=\rho\left(\left.h\right|_{A}\right)=\alpha$ and $\left.\gamma\right|_{\Gamma_{B}}=\rho\left(\left.h\right|_{B}\right)=\beta$. In other words, $\phi(\gamma)=\left(\left.\gamma\right|_{\Gamma_{A}},\left.\gamma\right|_{\Gamma_{B}}\right)=(\alpha, \beta)$, i.e., $\phi$ is surjective and therefore an isomorphism.

\section{REFERENCES}

[1] E. A. Kudryavtseva. Connected components of spaces of Morse functions with fixed critical points. Vestnik Moskov. Univ. Ser. I Mat. Mekh., (1):3-12, 2012.

[2] E. A. Kudryavtseva. The topology of spaces of Morse functions on surfaces. Math. Notes, 92(1-2):219-236, 2012. Translation of Mat. Zametki 92 (2012), no. 2, 241-261.

[3] E. A. Kudryavtseva. On the homotopy type of spaces of Morse functions on surfaces. Mat. Sb., 204(1):79-118, 2013.

[4] E. A. Kudryavtseva, D. A. Permyakov. Framed Morse functions on surfaces. Mat. Sb., 201(4):33-98, 2010.

[5] S. Maksymenko, B. Feshchenko. Orbits of smooth functions on 2-torus and their homotopy types. Matematychni Studii, 44(1):67-84, 2015. 
[6] S. Maksymenko, B. Feshchenko. Smooth functions on 2-torus whose kronrod-reeb graph contains a cycle. Methods Funct. Anal. Topology, 21(1):22-40, 2015.

[7] S. Maksymenko, A. Kravchenko. Automorphisms of Kronrod-Reeb graphs of Morse functions on compact surfaces. arXiv:1808.08746, 2018.

[8] Sergiy Maksymenko. Homotopy types of stabilizers and orbits of Morse functions on surfaces. Ann. Global Anal. Geom., 29(3):241-285, 2006.

[9] Sergiy Maksymenko. Functions with isolated singularities on surfaces. Geometry and topology of functions on manifolds. Pr. Inst. Mat. Nats. Akad. Nauk Ukr. Mat. Zastos., 7(4):7-66, 2010.

[10] Sergiy Maksymenko. Homotopy types of right stabilizers and orbits of smooth functions functions on surfaces. Ukrainian Math. Journal, 64(9):1186-1203, 2012.

[11] Sergiy Maksymenko. Deformations of functions on surfaces by isotopic to the identity diffeomorphisms. 2013.

[12] Stephen Smale. Diffeomorphisms of the 2-sphere. Proc. Amer. Math. Soc., 10:621-626, 1959.

Received: October 15, 2018, accepted: January 17, 2019.

Anna Kravchenko

Department of Geometry, Topology, and Dynamical Systems

Taras Shevchenko National University of Kyiv

Hlushrova Avenue, 4E, Kyiv, Ukraine, 03127

Email: annakravchenko1606@gmail.com

Sergiy Maksymenko

Topology Laboratory of Algebra and Topology Department

Institute of Mathematics of National Academy of Sciences of Ukraine

TereshChenkivs'KA STR. 3, Kyiv, Ukraine, 01024

Email: maks@imath.kiev.ua

ORCID: orcid.org/0000-0002-0062-5188 